\title{
Better by Design: The Potential of Design Thinking Approaches in Tackling the Widening Participation "Problem" in Higher Education
}

By:

Dr. Pauline Taylor

International concern relating to access and opportunity in Higher Education (Council of Europe, 1996; OECD, 2008; Rougaas, 2001) has generated increased research interest in how and why tertiary institutions tend to reproduce privilege (Bourdieu \& Passeron, 1977; Teese, 2011). The 'problem' of widening participation, particularly in relation to traditionally marginalized groups, has proven intractable despite concerted policy efforts (Tinto, 2006-7; Kift, Nelson \& Clarke, 2012). Theoretical work, such as that of Sellar and Gale (2011) and Dei (2008) highlights the need to move from a politics of representation to one of recognition. This paper draws on an analysis of data collected in the development and implementation of a policy and practice framework to support the transition to tertiary education at an Australian university. A design thinking approach was used to try and disrupt the dominant epistemologies and institutional practices that work as powerful technologies (Ball, 2003) in maintaining a dominant culture. Insights are provided into how and why institutional practices and epistemologies militate against inclusivity and ways in which institutions might design more positive and productive higher education experiences for increasingly diverse students are proposed.

Keywords: Design, Diversity, Epistemology

Stream: Design in Society 